\title{
Terre et agriculture sous la Révolution et l'Empire
}

Laurent Brassart, Jean-Pierre Jessenne, Peter M. Jones et Nadine Vivier

\section{(2) OpenEdition}

\section{Journals}

Édition électronique

URL : https://journals.openedition.org/ahrf/13567

DOI : $10.4000 /$ ahrf. 13567

ISSN : 1952-403X

Éditeur :

Armand Colin, Société des études robespierristes

Édition imprimée

Date de publication : 1 décembre 2015

Pagination : 145-155

ISBN : 9782200930028

ISSN : 0003-4436

\section{Référence électronique}

Laurent Brassart, Jean-Pierre Jessenne, Peter M. Jones et Nadine Vivier, «Terre et agriculture sous la Révolution et l'Empire », Annales historiques de la Révolution française [En ligne], 382 | octobre-décembre 2015, mis en ligne le 01 décembre 2018, consulté le 23 avril 2022. URL : http:// journals.openedition.org/ahrf/13567 ; DOI : https://doi.org/10.4000/ahrf.13567 
REGARDS CROISÉS

\title{
TERRE ET AGRICULTURE SOUS LA RÉVOLUTION ET L'EMPIRE
}

\author{
Laurent BRASSART \\ Jean-Pierre JESSENNE \\ Peter M. JONES \\ Nadine VIVIER
}

\section{Laurent Brassart ${ }^{1}$ \\ Des travaux récents sur la base d'analyses transnationales ont montré que beaucoup des événements agraires imputables à la}

(1) Débat modéré par Laurent Brassart, maître de conférences en histoire moderne à l'Université de Lille.

Jean-Pierre Jessenne est professeur émérite d'histoire moderne à l'Université de Lille, spécialiste du monde rural et de l'agriculture pendant la Révolution française et l'Empire. On lui doit notamment les ouvrages suivants : Pouvoir au village et Révolution : Artois (1760-1848), Presses universitaires de Lille, Lille, 1987 et Les campagnes françaises, entre mythe et histoire (XVIII ${ }^{e} X X I^{e}$ siècle), Paris, Armand Colin, 2006. Il a codirigé entre autres : L'Empire napoléonien. Une expérience européenne?, Paris, A. Colin, 2014 ; Clochemerle ou république villageoise? La conduite municipale des affaires villageoises en Europe, XVIII ${ }^{e}-X X^{e}$ siècle, Lille, Presses Universitaires du Septentrion, 2012 ; Les Élites rurales dans l'Europe médiévale et moderne, Toulouse, Presses Universitaires du Mirail, 2007.

Peter M. Jones est professeur émérite d'histoire de France à l'Université de Birmingham, spécialiste de l'histoire rurale de la Révolution française. Il est l'auteur de Politics and Rural Society : the Southern Massif Central, c. 1750-1880, Cambridge University Press (CUP), Cambridge, 1985 ; The Peasantry in the French Revolution, CUP, Cambridge, 1988 ; Liberty and Locality in Revolutionary France, 1760 1820 : Six Villages Compared, 1760-1820, CUP, Cambridge, 2003. Publication à venir : Agricultural Enlightement : Knowledge, Technology and Nature, 1750-1840, Oxford, Oxford University Press, 2016.

Nadine Vivier, professeure émérite d'histoire contemporaine à l'Université du Maine, est spécialiste du monde rural et de l'agriculture au XIX ${ }^{\mathrm{e}}$ siècle. Elle est l'auteure de Propriété collective et identité communale. Les biens communaux en France de 1750 à 1914, Publications de la Sorbonne, 1998 et a dirigé ou codirigé entres autres ouvrages sur ce sujet : Ruralités françaises et britanniques : approches comparées, PUR, Rennes, 2005 ; The State and Rural Societies. Policy and Education in Europe, 1750-2000, Brepols, Turnhout, 2008 ; Les systèmes cadastraux en Europe aux $19^{e}$ et $20^{e}$ siècles (avec Florence Bourillon), éditions du Comité pour l'Histoire Économique et Financière de la France, Paris, 2008 ; Élites et Progrès agricole, XVI ${ }^{e}-X X^{e}$ siècle, PUR, Rennes, 2009 ; Clochemerle ou république villageoise? La conduite municipale des affaires villageoises en Europe, XVIII ${ }^{e}-X X^{e}$ siècle, Presses Universitaires du Septentrion, Lille, 2012 
Révolution s'étaient déjà produits auparavant : c'est le cas de la sécularisation des biens du clergé (notamment avec le Joséphisme) ou de la remise en cause de certains droits de propriété. La Révolution peut-elle encore être tenue pour un moment déterminant dans l'histoire des campagnes européennes?

\section{Jean-Pierre Jessenne}

La Révolution doit être inscrite dans un mouvement européen, qui s'affirme dès les années 1760-1770, de libéralisation de l'économie agricole, y compris de l'accès à la terre, par exemple par les autorisations de clôture et la limitation des corvées au Danemark ou les mesures de restriction de la Mesta et de partage des terres communes dans l'Espagne de Charles III et Campomanes. La Révolution ne fait que catalyser - et c'est déjà beaucoup - ce processus et par conséquent tous les problèmes sociaux-politiques qui lui sont liés. En favorisant la prise de parole et les mouvements collectifs dès le début 1789, en suscitant les impatiences avec l'abolition d'abord partielle des droits seigneuriaux, en reposant la question de l'accès à la terre, la Révolution exacerbe les tensions économiques et sociales au sein des communautés rurales autant qu'elle accroît l'exigence de leur représentation politique. Avec la création des communes et des districts en décembre 1789, la Révolution ouvre une ère nouvelle et exemplaire dans la politisation et la représentation politique des ruraux, que le succès des premières élections municipales en 1790 confirme.

\section{Nadine Vivier}

Que ce soit pour la vente des biens ecclésiastiques ou le partage des communaux, la Révolution ne peut pas être tenue pour un régime novateur. Voire elle a, par ses hésitations législatives, plutôt freiné et parfois bloqué le processus de changement : la loi de partage des communaux du 10 juin 1793, qui comprend tout un volet sur la récupération des biens considérés par les municipalités comme usurpés, a engendré tant de procès que les députés l'ont suspendue en 1795 et que Bonaparte, pour apaiser les tensions, a stoppé le processus de vente ou de partage. En revanche, la Révolution est doublement déterminante - et à ce titre pleinement révolutionnaire lorsqu'elle abolit le régime féodal et accélère la transformation politique des campagnes.

\section{Peter M. Jones}

À partir des années 1750-1760, la montée des rivalités entre les grandes puissances européennes, d'une part, et l'augmentation des pressions 
fiscales, démographiques et philanthropiques au sein des états de l'autre, se sont articulées pour que nombre de dirigeants et d'administrations éclairés engagent des programmes de réformes agraires. Les premières initiatives en ce domaine sont à porter au crédit des états nordiques, en particulier les royaumes de Danemark-Norvège et de Suède. En 1789, les révolutionnaires français étaient aussi porteurs de grands projets de transformations agraires, même si les contraintes économiques et politiques en ont compromis la portée. Toutefois, les vestiges du féodalisme ont été liquidés bien plus rapidement en France que partout ailleurs en Europe, et ce grâce à la Révolution. Comme ils n'ont pas été rétablis, les rapports de forces politiques dans les campagnes en ont été durablement transformés en profondeur.

\section{Laurent Brassart}

Depuis Lecarpentier et Jaurès une vulgate s'est imposée : « la vente des biens nationaux fut l'événement le plus important de la Révolution française », notamment parce qu'elle aurait permis l'avènement d'une société bourgeoise et d'une société paysanne plus égalitaire, dont la petite exploitation familiale serait devenue la structure la plus saillante dans les campagnes ${ }^{2}$. Cette représentation doit-elle être corrigée aujourd'hui ?

\section{Jean-Pierre Jessenne}

Il faut à la fois corriger et relativiser cette représentation : d'une part seulement $10 \%$ environ des terres cultivables ont été vendues lors de la vente des biens nationaux de première origine; de l'autre, la répartition des biens vendus ne se traduit pas par l'avènement de la petite propriété paysanne mais seulement par sa consolidation ; enfin, la vente des biens nationaux ne s'est pas accompagnée d'une disparition de la grande propriété. Celle-ci a surtout reculé dans les quelques régions où l'emprise foncière monastique était forte (Cambrésis, Soissonnais notamment), ce qui représente une faible partie du territoire national.

(2) L'expression est celle de Georges Lecarpentier dans son ouvrage La vente des biens ecclésiastiques pendant la Révolution française, Paris, Alcan, 1908. Pour Jean Jaurès, la vente des biens nationaux révèle le caractère foncièrement bourgeois de la Révolution. Cf. Jean JAURÈs, Histoire socialiste de la Révolution française, Paris, 1901-1908.

Sur la question des biens nationaux, cf. Bernard BODINIER, « La vente des biens nationaux : essai de synthèse », AHRF, $\mathrm{n}^{\circ} 315,1999$, p. 7-19 et Bernard BODINIER et Éric TEYSSIER, L'événement le plus important de la Révolution. La vente des biens nationaux, Paris, CTHS/SER, 2000. 


\section{Nadine Vivier}

Jaurès était un homme de son temps et son jugement reflète ses engagements : ses positions anti-nobiliaires et hostiles aux communaux qu'il qualifie de « communisme de misère ». Homme du Sud-Ouest des petites exploitations familiales, il s'inscrit dans la lignée jacobine de l'idéal du petit producteur indépendant. Par conséquent, son jugement sur les biens nationaux exprime plus son idéal qu'une réalité historique.

\section{Jean-Pierre Jessenne}

La vente des biens nationaux a aussi constitué un élément fondamental de la politisation des ruraux en Révolution en mettant les questions de la propriété et de l'exploitation des terres au cœur des enjeux politiques.

\section{Nadine Vivier}

En effet, à certains égards, l'importance et l'impact de la vente des biens nationaux sont beaucoup plus politiques qu'économiques.

\section{Peter M. Jones}

Moi-aussi, je me demande dans quelle mesure la politique de vente des propriétés de l'Église, des émigrés et des corporations pendant la Révolution a vraiment fait entrer la société française dans le XIX ${ }^{\mathrm{e}}$ siècle, puisque la petite propriété foncière était déjà une réalité avant 1789 et que la grande propriété a persisté au-delà la Révolution. La véritable réussite de la Révolution tient en trois résultats : la libération du marché foncier, notamment en abolissant les contraintes seigneuriales, loin d'être faibles dans certaines régions ; la clarification de la notion de propriété ; la considération sociale accordée au petit propriétaire exploitant qui en retour suscita une réelle estime de lui-même. En ce sens, je crois que la Révolution a vraiment contribué au développement d'une « démocratie paysanne ».

\section{Laurent Brassart}

Des travaux récents, notamment ceux de Gérard Béaur, montrent que l'ouverture du marché foncier ordinaire est bien plus déterminante dans les comportements économiques des paysans que le marché extraordinaire des biens nationaux. Après la Révolution, pour nombre de paysans, la terre ne ferait plus l'objet d'un fétichisme mais s'échangerait facilement. Le rapport du paysan à sa terre a-t-il pu évoluer aussi rapidement et aussi profondément avec la Révolution ? 


\section{Peter M. Jones}

La focalisation des historiens universitaires sur le marché foncier exceptionnel des biens nationaux eut pour conséquence une certaine cécité de leur part à l'égard du marché ordinaire. Mon sentiment est que nous avons encore beaucoup à apprendre des mécanismes de ce marché et de ce qu'il révèle des attitudes des ruraux envers l'acquisition des terres. Même si j'admets la fluidité du marché foncier « ordinaire », bien identifié par Gérard Béaur et un certain nombre d'autres chercheurs, je me demande toutefois dans quelle mesure, la représentation qu'ils en font peut être généralisée ${ }^{3}$. Dans les régions françaises que je connais le mieux, notamment le Sud et l'Ouest, les occasions d'acquisition foncière étaient moindres et l'attachement culturel des paysans à la terre plus fort. En outre, dans de telles régions, des facteurs « institutionnels », comme l'impact du régime seigneurial sur le marché foncier, étaient plus prononcés. Du coup, j'hésite vraiment à faire de la marchandisation des terres un postulat et doute quelque peu de son applicabilité après la Révolution.

\section{Nadine Vivier}

Il faut sur cette question se garder de toute position tranchée. D'abord, au cours du premier $\mathrm{XIX}^{\mathrm{e}}$ siècle, le lien du paysan à sa terre se renforce face à la peur que les ultras inspirent. Même la loi sur le Milliard des Émigrés (27 avril 1825) ne parvient pas à dissiper au sein des paysanneries la double crainte d'une restitution à la noblesse des biens acquis à la Révolution et d'une hypothétique restauration des droits féodaux. Enfin, l'attachement du paysan à sa terre est aussi à relativiser en fonction de la taille de son exploitation : le petit paysan sous la Monarchie constitutionnelle est beaucoup plus intéressé à consolider sa propriété s'il veut rentabiliser et sécuriser les investissements sur son exploitation. Le risque des fermiers de se faire évincer par le propriétaire de leur exploitation au terme de baux de 3, 6, 9 ans est réel. En revanche, les plus riches exploitants, en particulier les gros fermiers, n'ont pas forcément d'intérêts économiques à accéder à la propriété, sauf peut-être à caresser l'ambition socio-politique d'accéder à la notabilité, ou au moins au droit d'éligibilité. Pour soutenir la réussite

(3) Gérard BÉAur, Phillipp SCHOFIELD, Jean-Michel ChEVET et Maria Teresa PÉREZ-PICAZO (eds.), Property Rights, Land Markets and Economic Growth in the European Countryside, Rural History in Europe, vol. 1, Turnhout, Brepols, 2013 ; Gérard BÉAUR, Le marché foncier à la veille de la Révolution. Les mouvements de propriété beaucerons dans les régions de Maintenon et de Janville de 1761 à 1790, Paris, Éditions de l'EHESS, 1984. 
économique de leur exploitation, il est plus intéressant pour eux d'investir dans des moyens de production que dans le marché foncier.

\section{Jean-Pierre Jessenne}

Deux autres éléments de relativisation s'imposent. D'une part, le marché ordinaire est incontestablement devenu plus ouvert depuis qu'avec la Révolution une partie des terres jadis neutralisées (en partie celles mainmortables) a perdu ce statut et a donc été vendue ; pour autant toutes les régions n'ont pas été concernées avec la même intensité que la Bourgogne par exemple. De l'autre, la propriété ne doit pas être regardée comme le seul critère de l'hégémonie économique et sociale : rappelons que la stratégie économique de la fermocratie consiste davantage à tenir sa terre en bail plus qu'à acquérir une propriété, afin de libérer de l'investissement dans les moyens de production (matériel et cheptels notamment). Finalement, l'événement le plus important pour la paysannerie française, après l'abolition du régime seigneurial, celui qui a le plus modifié en profondeur le rapport propriétaire/exploitant, a été l'adoption du statut du fermage en 1945. Les conditions de sécurité nécessaires à l'investissement d'un fermier sur son exploitation étaient enfin garanties.

\section{Laurent Brassart}

La Révolution eut-elle une véritable politique agraire ou s'estelle contentée de mesures de circonstances, plus inspirées par des préoccupations politiques et financières que sociales ?

\section{Peter M. Jones}

Les révolutionnaires de 1789 ont fait leur entrée dans l'arène politique avec un agenda agraire totalement utopique. Mais leur travail aussi long que vain pour élaborer un Code Rural entre 1789 et 1791 sonna le glas de leurs dernières illusions.

D'ailleurs, le dernier pan de leur législation agraire a été adopté dans l'urgence et sans débat sérieux, lors des dernières sessions de l'Assemblée nationale constituante. Ceci dit, un certain nombre de députés de la Convention nationale ont, quant à eux, vraiment porté des projets de réforme agraire contenant une dimension sociale réelle, c'est-à-dire égalitariste et redistributive. Mais, dans l'ensemble, ces projets n'ont jamais été exécutés, même si plusieurs représentants en mission ont essayé de résoudre la question de la faim de terre sur place. 


\section{Jean-Pierre Jessenne}

La Révolution n'a pas eu une politique agraire mais des politiques agraires qui ont considérablement varié selon les moments et les courants politiques. Deux grands temps sont à distinguer : d'abord celui de la Constituante et de la Législative (1789-août 1792), véritable moment d'imposition du libéralisme au monde rural, avec des mesures aussi emblématiques que le mode de vente des biens nationaux par corps d'exploitation d'un seul tenant et le rachat des droits féodaux ; ensuite celui de la «Révolution montagnarde » (juin 1793-juillet 1794), promotrice d'un changement agraire dans le sens d'un plus grand égalitarisme entre paysans. Pour autant, en ce qui concerne l'action des montagnards, il est difficile de parler d'un projet cohérent de politique agraire tant les contradictions en leur sein sont multiples. Après cette expérience, le Directoire revient à une politique plus libérale dans la lignée de celle de la Constituante et de la Législative.

\section{Nadine Vivier}

Le Directoire ne peut être réduit au seul retour triomphant au seul libéralisme. Sa politique agraire, notamment sur la question des biens communaux, a oscillé au gré des coups de balanciers politiques dont il était l'objet.

\section{Nadine Vivier et Jean-Pierre Jessenne}

De 1789 à 1799, au sein des différents régimes qui se sont succédés, jamais il n'y eut une politique agraire évidente et cohérente. Toutefois quelques continuités exceptionnelles peuvent être soulignées notamment à propos de la politique agraire que met en place François de Neufchâteau pendant le Directoire, après fructidor an $\mathrm{V}$, et qui reprend en partie ses idées avant-gardistes de 1792.

\section{Laurent Brassart}

L'historiographie ne s'est-elle pas trop cristallisée sur certains enjeux agraires (biens nationaux, biens collectifs, droits féodaux en particulier) au détriment d'autres agents de la transformation des campagnes comme la fiscalité, la hausse du salariat, la révolution juridique ? Ne sont-ce pas là les véritables agents durables du changement rural ? 


\section{Peter M. Jones}

L'approche des historiens de la Révolution de ma génération a été largement influencée par l'École des Annales. Il est désormais temps d'avancer, ou au moins de reformuler l'ordre du jour de la recherche en histoire agraire. Certains des thèmes traditionnels pourraient être rafraîchis en déplaçant la réflexion sur les questions de la taxation, de l'endettement y compris le désendettement, et des pratiques de succession. Mais nous devons également élargir notre angle de vision pour nous intéresser à la citoyenneté, à l'identité, au comportement électoral, aux niveaux de vie, à la consommation alimentaire des ruraux aussi bien qu'aux chaînes d'approvisionnement, à la science et à la technologie et la poursuite de l'objectif hors d'atteinte de l'égalité fiscale.

\section{Nadine Vivier et Jean-Pierre Jessenne}

Le problème n'est pas tant d'avoir minoré ou surestimé les enjeux agraires au détriment d'autres comme les transformations juridiques et fiscales, que la focalisation de l'historiographie sur la propriété pendant la Révolution. Emblématique de cette surestimation de la propriété, la belle étude sur le salariat agricole d'Yvonne Crebouw n'a pas eu le retentissement mérité, les travaux novateurs de Serge Aberdam sur le métayage n'ont pas eu de suite ${ }^{4}$. Quant aux grèves contre le Maximum en 1793-94, elles n'ont pas donné lieu à des études approfondies depuis Mathiez ${ }^{5}$.

\section{Jean-Pierre Jessenne}

Les vacances fiscales de 1789 au Directoire, la hausse de la rémunération des journaliers et saisonniers après l'entrée en guerre en 1792 sont certes importantes. Mais la liquidation progressive du système seigneurial du 4 août 1789 à l'an II l'est plus encore.

\section{Nadine Vivier}

On ne dira jamais combien, dans le milieu rural pendant la Restauration, grande est l'angoisse d'une restauration du régime seigneurial et d'une restitution des biens nationaux à leurs propriétaires de l'Ancien Régime. À ce titre, le projet d'un cadastre devient un enjeu autant juridique

(4) Yvonne CREBOUw, Salaires et salariés agricoles en France des débuts de la Révolution aux approches $d u X X^{e}$ siècle. Thèse soutenue sous la direction de Jean Bouvier, Université de Paris 1 , 1986 ; Serge ABERDAM, « La Révolution et les luttes des métayers » dans Études rurales, n 59, 1975 , p. 73-91.

(5) Albert MATHIEZ, La vie chère et le mouvement social sous la Terreur, Paris, Payot, 1927. 
que politique. Les paysanneries l'exigent pour consolider leurs propriétés face à la noblesse, quand les ultras le combattent vigoureusement. De même « les vacances fiscales» de 1789 au Consulat ont permis l'amélioration de la condition économique de nombre de paysans, d'autant que l'Ancien Régime finissant, s'il entendait agir pour plus d'égalité fiscale, cherchait aussi à perfectionner l'efficacité de l'administration fiscale comme l'a montré Mireille Touzery ${ }^{6}$.

\section{Laurent Brassart}

Vous semblez minorer l'importance du Code civil, cette « machine à hacher le sol »?

\section{Jean-Pierre Jessenne et Nadine Vivier}

Attention à ne pas focaliser l'historiographie sur la propriété et le mythe de l'égalité de la transmission!

\section{Peter M. Jones}

Comme notre connaissance du marché foncier avant comme après 1789 progresse, il est possible de mieux apprécier le rôle joué par le Code civil. En tout cas, il apparaît que, dans quelques régions, les ménages ruraux prenaient déjà en compte dans leurs stratégies de transmission la législation révolutionnaire sur les successions bien avant le Code civil de 1804.

\section{Nadine Vivier}

Certes le Code civil instaure l'égalité entre héritiers mais il comporte aussi nombre de dispositifs dérogatoires. Dans un volume d'Études Rurales, Joseph Goy a montré qu'il est un compromis génial qui autorise l'égalitarisme le plus strict aussi bien qu'un inégalitarisme très prononcé là où on avait l'habitude de le pratiquer et Pierre Lamaison, par une enquête auprès des notaires en 1980, a montré que ces pratiques locales ont perduré ${ }^{7}$.

(6) Mireille TOUZERY, « La dernière taille (Abolition des privilèges et technique fiscale d'après le rôle de Janvry pour les derniers mois de 1789 et pour 1790) », dans Histoire \& Mesure, 1997, vol. 12, $\mathrm{n}^{\circ} 1-2$, p. 193-142.

(7) Joseph GoY, « Transmission successorale et paysannerie pendant la Révolution française : un grand malentendu », dans Études rurales, vol. 110, n $110-112$, avril-décembre 1988, p. 45-56 ; Pierre LAMAISON, « La diversité des modes de transmission : une géographie tenace », dans Ibidem, p. 119-176. 


\section{Laurent Brassart}

La France de la Révolution conquiert une partie de l'Europe à partir de 1793 et impose son modèle. Quel est l'impact des transformations agraires de la Révolution française sur l'agriculture européenne?

\section{Nadine Vivier}

L'influence de la France sur une Europe qu'elle domine de 1794 à 1815 se fait davantage sur les structures agraires que sur l'imposition d'un modèle agricole. Le réseau international des agronomes était déjà constitué dès la fin de l'Ancien Régime avec des échanges très intenses ; ce qui explique que diverses expériences agraires étaient observées et discutées, notamment les modèles anglais, français, italiens en particulier ${ }^{8}$. Une fois encore, c'est en abolissant la féodalité que la Révolution française a exercé une influence déterminante sur l'Europe, selon trois configurations possibles : une influence directe, avec abolition de la féodalité sur la rive gauche du Rhin et la Westphalie par exemple ; une influence indirecte, notamment dans les régions et pays d'Europe centrale et orientale : dans ce cas, le succès de l'abolition de la féodalité dépendait de la capacité des gouvernements et des forces politiques à la rendre opérationnelle, ce qui explique son succès relatif ou partiel en Pologne et son échec en Hongrie ; enfin, les situations de refus de l'expérience française comme en Russie.

\section{Jean-Pierre Jessenne}

Un brouillard idéologico-historiographique recouvre la question des influences et des modèles agricoles. La comparaison usuelle entre un prétendu modèle agricole anglais performant, reposant sur de grandes exploitations capitalistes et l'innovation technique et agronomique, et un tout aussi prétendu modèle français de petites exploitations familiales autosubsistantes et archaïques est désormais battue en brèche par les historiens des deux côtés de la Manche. La plupart des pays européens combinent en fait des régimes agro-économiques variés selon les régions, les productions et les profils sociaux...

(8) Gilles Postel-VinAY et Maurice AYMARD, « La perception française de l'agriculture et de l'agronomie italiennes dans la première moitié du XIX ${ }^{\mathrm{e}}$ siècle », dans Roberto FINZI, Fra studio, politica ed economica : la societa agraria dalle origini all'età giolittiana, Bologna, 1992, p. 575-596. 


\section{Peter M. Jones}

Avec le passage de la défense de la Patrie à la réalisation de la Grande Nation en 1794-1795, il me semble que le régime révolutionnaire a renoncé à tout projet de transformation agraire au sein de ses frontières originelles pour privilégier l'imposition de ses principales réformes juridiques, en particulier la saisie et la vente des biens de l'Église, aux territoires « libérés/occupés ». Cette politique de la Révolution française dans les territoires qu'elle occupe a provoqué par contrecoup une réaction des monarchies dont les élites éclairées envisageaient encore avant la guerre une réforme agraire : face aux dangers politiques et sociaux d'une telle réforme et face à la menace française, certaines ont préféré renoncer, d'autres au contraire la poursuivre. Ce fut le cas, par exemple, du royaume de Danemark-Norvège. En ce sens, on peut donc dire que la Révolution française a constitué autant une source d'inspiration qu'un repoussoir pour les États qui envisageaient une réforme agraire.

Laurent BRASSART

Maître de conférence en histoire moderne

Université de Lille 3

IRHIS UMR 8529

Jean-Pierre JESSENNE Professeur émérite d'histoire moderne à l'Université de Lille III jean-pierre.jessenne@wanadoo.fr Peter M. JONES Professeur émérite Departement of History, School of History and Cultures University of Birmingham p.m.jones@bham.ac.uk

Nadine VIVIER

Professeur émérite d'histoire moderne à l'Université du Maine nadine.vivier@univ-lemans.fr 